\title{
Antiviral Effect of Korean Red Ginseng Extract and Ginsenosides on Murine Norovirus and Feline Calicivirus as Surrogates for Human Norovirus
}

\author{
Min Hwa Lee ${ }^{1}$, Bog-Hieu Lee ${ }^{1,2}$, Ji-Youn Jung ${ }^{3}$, Doo-Sung Cheon ${ }^{4}$, Kyung-Tack Kim ${ }^{5}$, and \\ Changsun Choi ${ }^{12^{*}}$ \\ ${ }^{1}$ Department of Food and Nutrition, Graduate School, Chung-Ang University, Seoul 156-756, Korea \\ ${ }^{2}$ School of Food Science and Technology, College of Natural Science, Chung-Ang University, Anseong 456-756, Korea \\ ${ }^{3}$ Department of Companion and Laboratory Animal Science, College of Industrial Science, Kongju National University, \\ Yesan 340-702, Korea \\ ${ }^{4}$ Divison of Enteric and Hepatitis Viruses, Korea Centers for Disease Control and Prevention, Osong 363-951, Korea \\ ${ }^{5}$ Korea Food Research Institute, Seongnam 463-746, Korea
}

Korean red ginseng has been studied various biological activities such as immune, anti-oxidative, anti-microbial, and anticancer activities but antiviral mechanism needs further studies. In this study, we aimed to examine the antiviral effects of Korea red ginseng extract and ginsenosides on norovirus surrogate, including murine norovirus (MNV) and feline calicivirus (FCV). We evaluated the pre-, co-, and post-treatment effects of Korean red ginseng (KRG), ginsenosides $\mathrm{Rb}_{1}$ and $\mathrm{Rg}_{1}$. To measure the antiviral effect and cytotoxicity of KRG extract, and ginsenosides $\mathrm{Rb}_{1}$ and $\mathrm{Rg}_{1}$, we treated Crandell-Reese Feline Kidney for FCV or RAW264.7 cells for MNV with concentrations of $0,5,6.7,10,20 \mathrm{ug} / \mathrm{mL}$ total saponin. There was cytotoxic effect in the highest concentration $20 \mathrm{ug} / \mathrm{mL}$ of KRG extract so this concentration was excluded in this study. The FCV titer was significantly reduced to $0.23-0.83 \log _{10} 50 \%$ tissue culture infectious dose $\left(\mathrm{TCID}_{50}\right) / \mathrm{mL}$ in groups pre-treated with red ginseng extract or ginsenosides. The titer of MNV was significantly reduced to $0.37-1.48 \log _{10} \mathrm{TCID}_{50} / \mathrm{mL}$ in groups pre-treated with red ginseng extract or ginsenosides. However, there was no observed antiviral effect in groups co-treated or post-treated with KRG and its constituents. Our data suggest that KRG extract has an antiviral effect against norovirus surrogates. The antiviral mechanisms of KRG and ginsenosides should be addressed in future studies.

Keywords: Panax ginseng, Antiviral activity, Murine norovirus, Feline calicivirus, Ginsenosides

\section{INTRODUCTION}

Norovirus (NoV) is a well-known foodborne viral pathogen that causes acute gastroenteritis worldwide. NoVs contribute to more than $50 \%$ of foodborne outbreaks [1] and NoV cases have significantly increased in Korea since 2000 . NoV outbreaks in school frequently

(c) This is an Open Access article distributed under the terms of the Creative Commons Attribution Non-Commercial License (http://creativecommons.org/licenses/by-nc/3.0/) which permits unrestricted non-commercial use, distribution, and reproduction in any medium, provided the original work is properly cited. caused socio-economic loss in Korea from 2002 to 2006. We reported the seasonal prevalence of asymptomatic NoV infection in children in Korea [2]. NoV is transmitted by foodborne, water-borne, person-to-person contact, and airborne mechanisms. Asymptomatic carriers are

Received 07 Jul. 2011, Revised 22 Aug. 2011, Accepted 22 Aug. 2011

*Corresponding author
E-mail: cchoi@cau.ac.kr
Tel: +82-31-670-4589, Fax: +82-31-676-8741 
also suspected as an important source of NoV infection $[2,3]$.

The clinical symptoms of NoV infection include diarrhea, vomiting, and abdominal cramping, which lasts 3 to $4 \mathrm{~d}$. In particular, NoV-related viral gastroenteritis in young children, elders, and immunocompromised people can cause severe symptoms. Mortality due to NoV infection in developing countries is higher than in developed countries [3]. However effective vaccines and medicines for the prevention and treatment of NoV infection have not been developed [4]. Only food safety and the personal hygiene of the food handler can prevent the outbreak or sporadic infection of NoV [5].

Because human NoV cannot be cultivated in a cell culture system, NoV surrogates are used to investigate the efficacy of chemical disinfectants. Feline calicivirus (FCV) and murine norovirus (MNV), family Caliciviridae, have similar physical characteristics, genome organization, and replication strategies to those of human NoV [4,6-8]. Therefore, antiviral or virucidal activity of natural and synthetic compounds are typically tested using FCV and MNV-1 models [3,4,6,7].

Ginseng (Panax ginseng) is the most well-known oriental herbal medicine in Asian countries including Korea, China, and Japan. Anti-oxidative, anti-microbial, and anti-cancer activities of $P$. ginseng are the well-known beneficial effects in oriental medicine [9-11]. The antiviral activities of $P$. ginseng have also been reported in several previous studies $[9,11,12]$. Although it has been reported that Panaxagin from $P$. ginseng possesses antifungal and antiviral activities [9], the mechanisms underlying the antiviral activity have not yet been elucidated. Therefore, we aimed to investigate the antiviral activities of Korean red ginseng (KRG) extract against NoV surrogates.

\section{MATERIALS AND METHODS}

\section{Viruses and cell lines}

Crandell Reese feline kidney (CRFK) cells, RAW 264.7 cells, and a FCV-F9 strain were purchased from ATCC (Manassas, VA, USA). MNV was kindly provided by Dr. Skip Virgin (Washington University, St. Louis, MO, USA). CRFK and RAW 264.7 cell lines were maintained at $37^{\circ} \mathrm{C}$ in $5 \% \mathrm{CO}_{2}$. Viral stocks of MNV and $\mathrm{FCV}$ have previously been described $[6,7]$.

\section{Red ginseng extract and ginsenosides}

KRG extract was provided by the Korea Ginseng Corporation (Daejeon, Korea). The ginseng content of the KRG extract was $85.6 \mathrm{mg} / \mathrm{g}$ and the water content was $36.7 \%$. Among the crude saponins, the total content of $\mathrm{Rg}_{1}$ and $\mathrm{Rb}_{1}$ was $4.5 \mathrm{mg} / \mathrm{g}$. Purified ginsenoside $R b_{1}$ (catalog no. ASB-00007191-005) and $\mathrm{Rg}_{1}$ (catalog no. ASB-00007221-005) were purchased from Chromadex (Irvine, CA, USA) and re-dissolved into distilled water.

\section{Antiviral effect of Korean red ginseng extract or purified ginsenoside}

To investigate the antiviral effect of $\mathrm{KRG}$ and ginsenosides against FCV and MNV, we used the $50 \%$ tissue culture infectious dose $\left(\mathrm{TCID}_{50}\right)$ assay with several concentrations of KRG or purified ginsenoside at pre-, co-, and post-virus infection.

\section{Pre-treatment with Korean red ginseng or ginsen- osides prior to virus infection}

Ninety six-well cell culture plates were seeded with $2 \times 10^{4}$ of CRFK or RAW 264.7 cell lines per well. After $24 \mathrm{~h}$, cell media was removed and washed three times with phosphate buffered saline (PBS). CRFK and RAW 264.7 cell lines were treated for $24 \mathrm{~h}$ with $0,5.0,6.7$, 10.0 , and $20.0 \mathrm{ug} / \mathrm{mL}$ total saponin of $\mathrm{KRG}$ extract or ginsenosides respectively for each of the 96-well plate. Each of the KRG extract or ginsenosides was diluted in Dulbecco's minimum Eagles medium (DMEM). Then, the KRG extract or ginsenoside was removed from the 96-well plate and the cells were washed once with PBS. The CRFK and RAW 264.7 cells were then inoculated with 10-fold serial dilutions of FCV or MNV from $10^{\circ}$ to $10^{-10}$. Viral absorption was performed on a shaking incubator at $80 \mathrm{rpm}$ for $90 \mathrm{~min}$. Finally, $100 \mathrm{uL}$ DMEM was added to each well of a 96-well plate. The cytopathic effect was examined under a microscope for up to $5 \mathrm{~d}$ [13]. Negative control was no treated with KRG extract or ginsenosides, and positive control was no inoculated with FCV or MNV. Viral titration was 4.89-5.50 $\log _{10} \mathrm{TCID}_{50}$ $\mathrm{mL}$ of FCV and 6.58-6.91 $\log _{10} \mathrm{TCID}_{50} / \mathrm{mL}$ of MNV.

\section{Co-treatment with Korean red ginseng or ginsen- osides and virus}

As with the pre-treatment experimental model, $2 \times 10^{4}$ of CRFK or RAW 264.7 cells were seeded in a 96-well plate. After CRFK or RAW 264.7 cells were monolayered for $24 \mathrm{~h}$, the DMEM was removed and the cells were washed one time with PBS. The total saponin of KRG extract or purified ginsenoside was prepared in DMEM at concentrations of $0.0,5.0,6.7,10.0$, and $20.0 \mathrm{ug} / \mathrm{mL}$. The mixture of surrogate virus and KRG or purified ginsenoside was serially diluted and then used to inoculate the cells. Viral absorption was performed on a shaking incu- 
bator at $80 \mathrm{rpm}$ for $90 \mathrm{~min}$. Each well was filled with 100 uL DMEM and the cells were observed for five days after infection. Viral titration was 4.89-5.50 $\log _{10} \mathrm{TCID}_{50} / \mathrm{mL}$ of FCV and 6.58-6.91 $\log _{10} \mathrm{TCID}_{50} / \mathrm{mL}$ of MNV.

\section{Post-treatment with Korean red ginseng or gin- senosides after virus infection}

Ten-fold diluted FCV or MNV was prepared and added to the wells containing CRFK or RAW 264.7 cell monolayers. Viral inoculation and incubation was performed in the same way as with the pre-treatment model. At 18 to $24 \mathrm{~h}$ post-virus inoculation, the cell media was removed and washed with PBS. The virus-infected cell layers were treated with $0.0,5.0,6.7,10.0$, and $20.0 \mathrm{ug} /$ $\mathrm{mL}$ total saponin of KRG or ginsenoside. The cytopathic effect was examined daily for $5 \mathrm{~d}$. Viral titration was 4.89-5.50 $\log _{10} \mathrm{TCID}_{50} / \mathrm{mL}$ of FCV and 6.58-6.91 $\log _{10} \mathrm{~T}$ $\mathrm{CID}_{50} / \mathrm{mL}$ of $\mathrm{MNV}$.

\section{Statistical analysis}

Triplicate data were obtained for all the experimental groups. The statistical analysis for the treatments and controls was performed using ANOVA and Duncan's multiple range test with SAS ver. 9.2 (SAS Institute, Cary, NC, USA).

\section{RESULTS}

\section{Effect of Korean red ginseng or ginsenoside pre- treatment on norovirus surrogates}

The mean titers of FCV and MNV were $5.2 \pm 0.3 \log _{10}$ $\mathrm{TCID}_{50} / \mathrm{mL}$ and $6.7 \pm 0.2 \log _{10} \mathrm{TCID}_{50} / \mathrm{mL}$ respectively. The pre-treatment effects of KRG on NoV surrogates are shown in $1 \mathrm{~A}$ and $1 \mathrm{~B}$. In CRFK cells pretreated with 5 , 6.7, and $10 \mathrm{ug} / \mathrm{mL}$ of $\mathrm{KRG}$ total saponin, the titer of FCV was reduced to $0.13 \pm 0.51,0.42 \pm 0.42$ and $0.83 \pm 0.36$ log, respectively. Especially, $10 \mathrm{ug} / \mathrm{mL}$ of $\mathrm{KRG}$ total saponin group differed significantly compared with control group ( $p<0.01)$. In RAW 264.7 cells pretreated with 5, $6.7,10 \mathrm{ug} / \mathrm{mL}$ of total KRG saponin, the titer of MNV was reduced to $0.38 \pm 0.41,0.73 \pm 0.19$ and $1.48 \pm 0.27 \mathrm{log}$ significantly $(p<0.01)$. Although a cytotoxic effect was observed in the CRFK and RAW 264.7 cells treated with $20 \mathrm{ug} / \mathrm{mL}$ KRG extract, cell death or damage was not found in CRFK and RAW 264.7 treated with less than 20 $\mathrm{ug} / \mathrm{mL}$ of $\mathrm{KRG}$ total saponin extract or purified ginsenosides.

Pre-treatment of CRFK and RAW 264.7 cells with purified ginsenoside $\mathrm{Rg}_{1}$ significantly reduced the titer of FCV and MNV, as shown in 2A and 2B. Treatment with
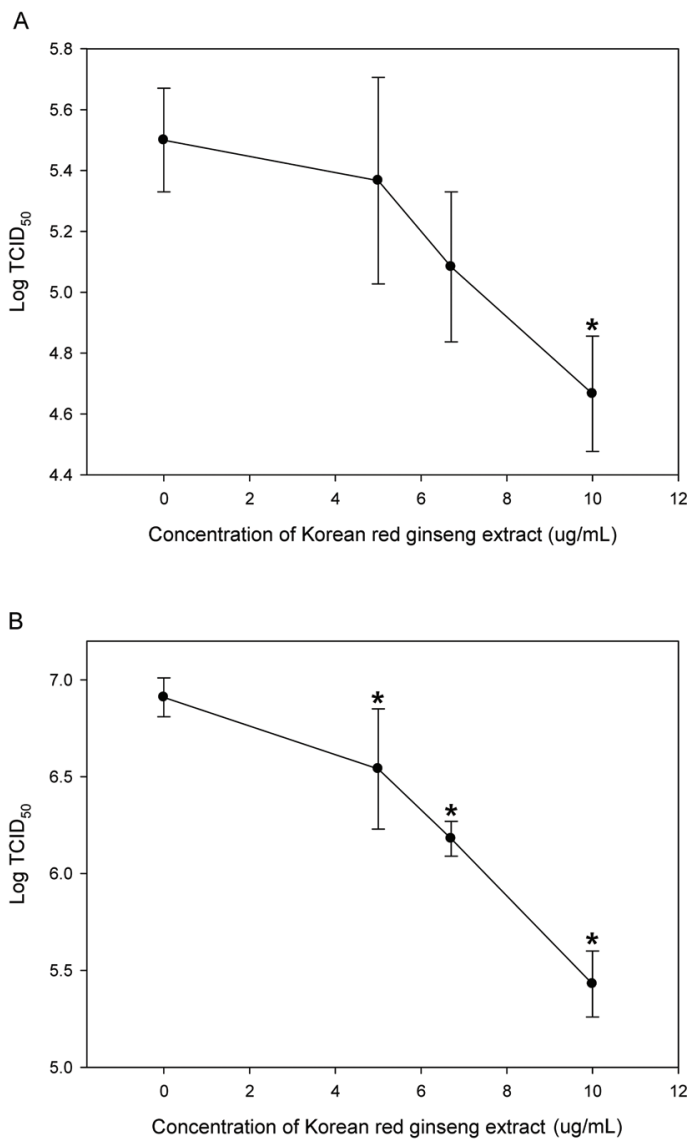

Fig. 1. Effect of ginseng extract. (A) Titration of feline calicivirus on Crandell Reese feline kidney cells pretreated with Korean red ginseng extract for $24 \mathrm{~h}$. (B) Titration of murine norovirus on RAW264.7 cells pretreated with Korean red ginseng extract for $24 \mathrm{~h}$. Values are mean $\pm S D$. The symbol * is used to indicate statistical significance with $p<0.01$. $T C I D_{50}$, tissue culture infectious dose.

more than $5 \mathrm{ug} / \mathrm{mL}$ of the ginsenoside $\mathrm{Rg}_{1}$ resulted in a significant reduction in FCV and MNV titers $(p<0.01)$. In CRFK cells pretreated with 5, 6.7, 10, and $20 \mathrm{ug} / \mathrm{mL}$ ginsenoside $\mathrm{Rg}_{1}$, the titer of FCV was reduced to $0.29 \pm 0.41$, $0.26 \pm 0.37,0.19 \pm 0.48$, and $0.43 \pm 0.57 \mathrm{log}$, respectively $(p<0.01)$. Pretreated with ginsenoside $\mathrm{Rg}_{1}$ in Raw 264.7 cell, the titer of MNV was reduced significantly in all of the area. Pretreated with 5, 6.7, 10, and $20 \mathrm{ug} / \mathrm{mL}$ of the ginsenoside $\mathrm{Rg}_{1}$, the titer of $\mathrm{MNV}$ was reduced to $0.61 \pm 0.39,0.64 \pm 0.32,1.21 \pm 0.37$ and $0.99 \pm 0.22 \log$, respectively $(p<0.01)$.

Pre-treatment of CRFK and RAW 264.7 cells with purified ginsenoside $R b_{1}$ significantly reduced the titers of FCV and MNV, as shown in Fig. 3A and 3B $(p<0.01)$. In CRFK cell pretreated with 5, 6.7, 10, and $20 \mathrm{ug} / \mathrm{mL}$ ginsenoside $\mathrm{Rb}_{1}$, the titer of FCV was reduced to $0.32 \pm 0.19$, $0.39 \pm 0.19,0.23 \pm 0.28$, and $0.33 \pm 0.29 \mathrm{log}$, respectively $(p<0.01)$. In RAW 264.7 cells pretreated with 5, 6.7, and 

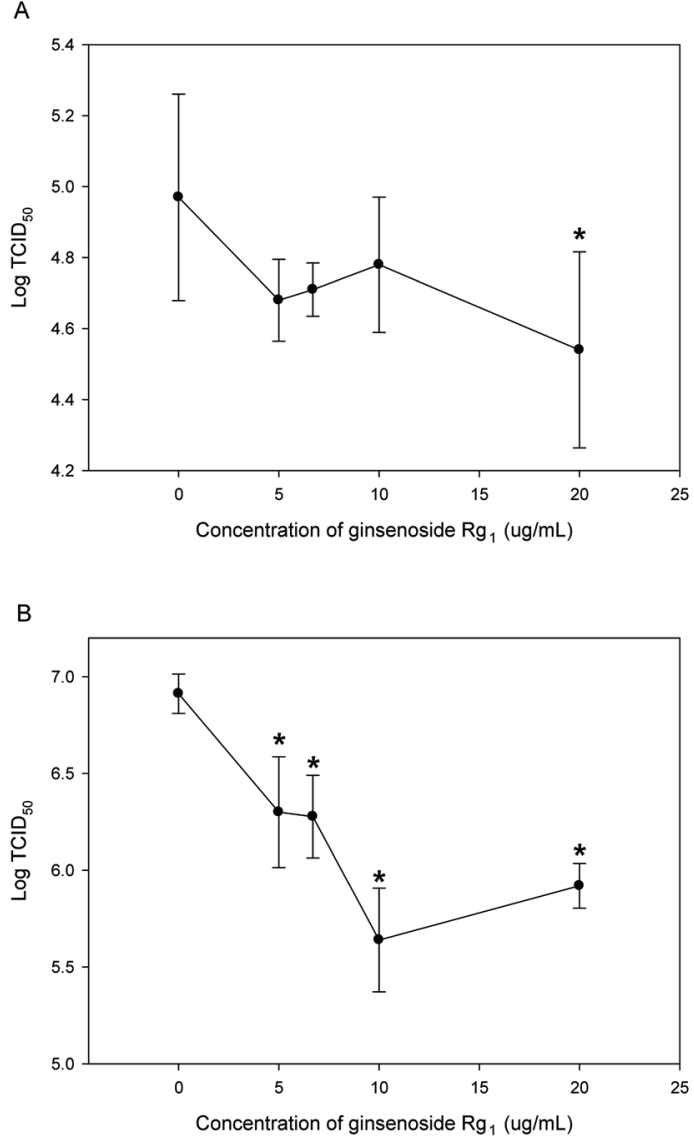

Fig. 2. Effect of purified ginsenoside $\mathrm{Rg}_{1}$. (A) Titration of feline calicivirus on Crandell Reese feline kidney cells pretreated with purified ginsenoside $\mathrm{Rg}_{1}$ for $24 \mathrm{~h}$. (B) Titration of murine norovirus on RAW264.7 cells pretreated with purified ginsenoside $\mathrm{Rg}_{1}$ for $24 \mathrm{~h}$. Values are mean $\pm S D$. The symbol * is used to indicate statistical significance with $p<0.01$. $\operatorname{TCID}_{50}$, tissue culture infectious dose.

$10 \mathrm{ug} / \mathrm{mL}$ ginsenoside $\mathrm{Rb}_{1}$, the titer of MNV was significantly reduced to $0.38 \pm 0.09,0.36 \pm 0.28$, and $0.41 \pm 0.25$ $\log$, respectively. However, there was no statistically significant difference in MNV titer for the $20 \mathrm{ug} / \mathrm{mL} \mathrm{Rb}_{1}$ treatment groups.

\section{Effect of co-treatment with Korean red ginseng or ginsenoside on norovirus surrogates}

There was no significant antiviral activity against FCV in CRFK cells co-treated with various concentrations of $\mathrm{KRG}$, or ginsenosides $\mathrm{Rb}_{1}$ and $\mathrm{Rg}_{1}$. FCV titers were $4.81 \pm 0.34$ to $5.36 \pm 0.06 \log _{10} \mathrm{TCID}_{50} / \mathrm{mL}$ in the KRG group, $3.49 \pm 0.62$ to $3.89 \pm 0.19 \log _{10} \mathrm{TCID}_{50} / \mathrm{mL}$ in the $\mathrm{Rb}_{1}$ group, and $3.71 \pm 0.08$ to $3.93 \pm 0.12 \log _{10} \mathrm{TCID}_{50} /$ $\mathrm{mL}$ in the $\mathrm{Rg}_{1}$ group. Antiviral activity was not observed against MNV when cells were co-treated with various concentrations of KRG, or ginsenosides $\mathrm{Rb}_{1}$ and $\mathrm{Rg}_{1}$ (data not shown).
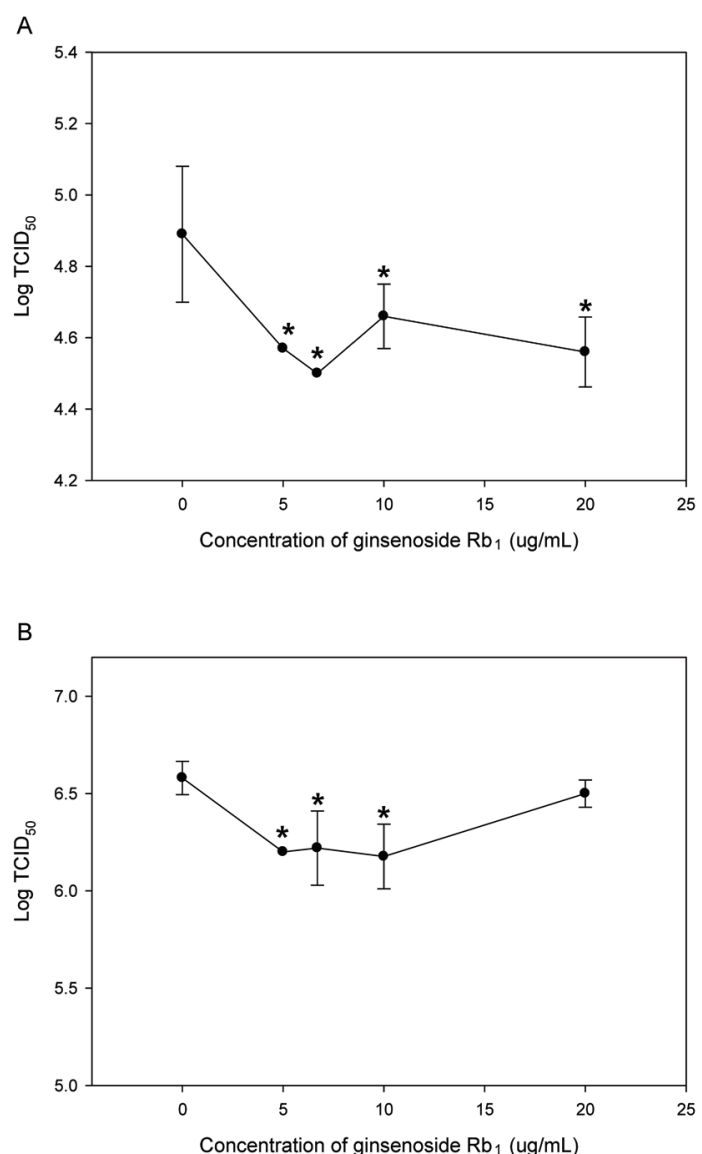

Fig. 3. Effect of purified ginsenoside $R b_{1}$. (A) Titration of feline calicivirus on Crandell Reese feline kidney cells pretreated with purified ginsenoside $R b_{1}$ for $24 \mathrm{~h}$. (B) Titration of murine norovirus on RAW264.7 cells pretreated with purified ginsenoside $R b_{1}$ for $24 \mathrm{~h}$. Values are mean $\pm S D$. The symbol ${ }^{*}$ is used to indicate statistical significance with $p<0.01$. $\operatorname{TCID}_{50}$, tissue culture infectious dose.

\section{Effect of post-treatment with Korean red ginseng or ginsenoside on norovirus surrogates}

There were no significant differences in titers of FCV for CRFK cells post-treated with KRG, or the ginsenosides $\mathrm{Rb}_{1}$ and $\mathrm{Rg}_{1}$. FCV titers were $4.50 \pm 0.07$ to 4.70 $\pm 0.26 \log _{10} \mathrm{TCID}_{50} / \mathrm{mL}$ in the KRG group, $4.22 \pm 0.19$ to $4.11 \pm 0.19 \log _{10} \mathrm{TCID}_{50} / \mathrm{mL}$ in the $\mathrm{Rb}_{1}$ group, and $4.25 \pm 0.23$ to $4.40 \pm 0.06 \log _{10} \mathrm{TCID}_{50} / \mathrm{mL}$ in the $\mathrm{Rg}_{1}$ group. There was no significant inactivation of MNV in RAW 264.7 cells post-treated with KRG, or the ginsenosides $\mathrm{Rb}_{1}$ and $\mathrm{Rg}_{1}$ (data not shown).

\section{DISCUSSION}

In order to investigate antiviral activity of KRG extract, we analyzed cells pre-treated, co-treated and posttreated with KRG extract or purified ginsenosides (Figs. $1-3)$. It was very interesting that a significant reduction in 
NoV surrogates was observed only in groups pre-treated with KRG (Fig. 1A, B). In contrast, the viral titers of FCV or MNV were not affected in the groups co-treated or post-treated with KRG. This result suggests that pretreatment with KRG triggers an antiviral environment against NoV surrogates.

Sung et al. [11] reported that the administration of KRG increases the CD4 T-cell counts and reduces the mutation rate of the human immunodeficiency virus (HIV) genome in HIV type 1 infected patients. Cho et al. [12] reported a significant reduction in HIV p24 secretion in an in vivo culture model treated with KRG. The 26$\mathrm{kDa}$ panaxagin protein identified in $P$. ginseng has ribonuclease activity that has an inhibitory effect on HIV reverse transcriptase, as well as antifungal activity against Coprinus comatus and Fusarium oxysporum [9]. To our knowledge, the antiviral activity of KRG against human NoV has not been reported because of the lack of a suitable cell culture system. Based on our data, pre-treatment with KRG may interfere with the replication of the $\mathrm{NoV}$ surrogates, FCV and MNV. However, the antiviral activity of KRG against human NoV should be evaluated in further studies because viral titers were reduced more in the MNV than in the FCV model.

In this study, the antiviral activity of ginsenoside $\mathrm{Rg}_{1}$ was stronger than that of ginsenoside $\mathrm{Rb}_{1}$ in both the FCV and MNV models. Treatment with ginsenoside $\mathrm{Rg}_{1}$ reduced both FCV and MNV titers, but the titer of MNV was reduced more than that of FCV (Fig. 2A, B). Ginsenoside $\mathrm{Rb}_{1}$ reduced the FCV titer in a dose-dependent fashion. Ginsenoside $\mathrm{Rb}_{1}$ significantly inhibited the MNV titer except $20 \mathrm{ug} / \mathrm{mL}$ of dose (Fig. 3A, B). Interestinlgy, reduction of MNV titer in $20 \mathrm{ug} / \mathrm{mL}$ treatment of $\mathrm{Rb}_{1}$ and $\mathrm{Rg}_{1}$ showed slightly less than in $10 \mathrm{ug} / \mathrm{mL}$ of $\mathrm{Rb}_{1}$ or $\mathrm{Rg}_{1}$. Unlike general dose response reaction, the characteristics of hormesis is a low dose beneficial effect and a high dose toxic effect [14]. Thus, these phenomena may explain the reduced antiviral activity of $\mathrm{Rb}_{1}$ or $\mathrm{Rg}_{1}$. Our observations are supported by the finding that MNV and FCV have different resistances against chemical disinfectants and antiviral food components including pomegranate and proanthocyanidin $[4,15,16]$. As MNV is known to be resistant to most treatment conditions, the antiviral effect of both ginsenoside $\mathrm{Rg}_{1}$ and $\mathrm{Rb}_{1}$ was more significant in the FCV model compared to the MNV model. However, the antiviral effect of KRG extract was equally strong in both models, suggesting that other ingredients in the KRG extract may have a synergistic effect on antiviral activity.

Like KRG extract, pomegranate extract has been shown to inhibit the infectivity of NoV surrogate models and other human viruses [4]. High levels of polyphenols, anthocyanins, and glycosides in pomegranate extract have been proposed to be antiviral ingredients $[4,17]$. Among the flavonoid compounds of pomegranate, punicalagin is reported to be a potent water-soluble antioxidant that inhibits influenza virus [17]. In addition to pomegranate juice, it has been reported that cranberry juice, grape juice, and orange juice lower the titer of FCV, MNV and MS2 (ssRNA) bacteriophage [18,19]. This phenomenon is thought to be due to alterations in the viral capsid or inhibition of binding to the host cell receptors $[4,16,17]$.

The food glycome has been proposed to inhibit the adhesion of pathogenic microorganisms [20]. In particular, anti-adhesive carbohydrates inhibit the adhesion of microbial toxins or enteric pathogens including bacteria, virus and protozoa [21]. Recently, pectin-type polysaccharides and arabinoglactan identified in $P$. ginseng were reported to have anti-adhesive activities against pathogenic bacteria [22]. Because surface lectins of the influenza virus are used to adhere to host receptors, some carbohydrates can interfere with virus adhesion by competing for binding sites [21]. It has been reported that blood type antigen receptors are used for the attachment of human NoV [2], so some carbohydrates in KRG may reduce the viral infectivity of NoV surrogates through an anti-adhesive effect.

The active substances of ginseng or KRG are the ginsenosides, which are distributed from the root to the stem and leaves of ginseng. Among the ginsenosides, $\mathrm{Rb}_{1}$ and Re have successfully been used as vaccine adjuvants to enhance the immune response $[23,24]$. The effect of ginsenoside $\mathrm{Rb}_{1}$ adjuvant in porcine parvovirus vaccine enhances the humoral and cellular immunity [23]. Similar to ginsenoside $\mathrm{Rb}_{1}$, ginsenoside $\mathrm{Re}$ also induces the activation of Th1 and Th2 lymphocytes and produces antiviral cytokines against influenza virus [24]. Both studies demonstrate the enhanced production of interferon gamma and antigen specific immunoglobulins (Igs) G1, $\mathrm{IgG}_{2 \mathrm{a}}$, and $\mathrm{IgG}_{2 \mathrm{~b}}$. Thus, the antiviral activities of KRG or ginsenosides fractions likely involve direct inhibition against viral particles or host cell receptors as well as immune enhancement. Although $\mathrm{Rb}_{1}$ did not show strong antiviral activity against the MNV model, the immunological effects of the $\mathrm{Rb}_{1}$ fraction against $\mathrm{NoV}$ need to be determined in further studies.

In conclusion, we found that pre-treatment with $\mathrm{KRG}$, and ginsenoside $\mathrm{Rg}_{1}$ and $\mathrm{Rb}_{1}$ significantly inhibits $\mathrm{FCV}$ and MNV dose-dependently. Although we did not in- 
vestigate the antiviral mechanisms of KRG or ginsenosides, the reduced infectivities of the NoV surrogates are thought to be due to the inhibition of adhesion to cell receptors or the formation of antiviral environments. Interestingly, co-treatment or post-treatment with KRG and the ginsenosides $\mathrm{Rg}_{1}$ and $\mathrm{Rb}_{1}$ were not effective at inhibiting FCV and MNV infectivity in the in vitro models. However, it is possible that co-treatment or posttreatment with KRG or ginsenosides would be effective at inhibiting NoV in vivo because some ginsenosides inhibit the virus by enhancing humoral and cellular immunity. Therefore, the antiviral mechanisms of KRG and ginsenosides need to be elucidated and active substances should be identified in further studies.

\section{ACKNOWLEDGEMENTS}

The study was supported by a grant from the Korean Society of Ginseng funded by the Korea Ginseng Corporation in 2009.

\section{REFERENCES}

1. Patel MM, Hall AJ, Vinje J, Parashar UD. Noroviruses: a comprehensive review. J Clin Virol 2009;44:1-8.

2. Cheon DS, Jeong HS, Jeong A, Lee KB, Lee MH, Tahk $\mathrm{H}$, Choi C. Seasonal prevalence of asymptomatic norovirus infection in Korean children. Foodborne Pathog Dis 2010;7:1427-1430.

3. Yoon JS, Lee SG, Hong SK, Lee SA, Jheong WH, Oh SS, Oh MH, Ko GP, Lee CH, Paik SY. Molecular epidemiology of norovirus infections in children with acute gastroenteritis in South Korea in November 2005 through November 2006. J Clin Microbiol 2008;46:1474-1477.

4. Su X, Sangster MY, D'Souza DH. In vitro effects of pomegranate juice and pomegranate polyphenols on foodborne viral surrogates. Foodborne Pathog Dis 2010;7:1473-1479.

5. Koopmans M, Duizer E. Foodborne viruses: an emerging problem. Int J Food Microbiol 2004;90:23-41.

6. Fino VR, Kniel KE. UV light inactivation of hepatitis A virus, Aichi virus, and feline calicivirus on strawberries, green onions, and lettuce. J Food Prot 2008;71:908-913.

7. Wei J, Jin Y, Sims T, Kniel KE. Survival of murine norovirus and hepatitis A virus in different types of manure and biosolids. Foodborne Pathog Dis 2010;7:901-906.

8. Wobus CE, Thackray LB, Virgin HW 4th. Murine norovirus: a model system to study norovirus biology and pathogenesis. J Virol 2006;80:5104-5112.

9. Ng TB, Wang H. Panaxagin, a new protein from Chinese ginseng possesses anti-fungal, anti-viral, translation-inhibiting and ribonuclease activities. Life Sci 2001;68:739749.

10. Sung WS, Lee DG. The combination effect of Korean red ginseng saponins with kanamycin and cefotaxime against methicillin-resistant Staphylococcus aureus. Biol Pharm Bull 2008;31:1614-1617.

11. Sung H, Jung YS, Cho YK. Beneficial effects of a combination of Korean red ginseng and highly active antiretroviral therapy in human immunodeficiency virus type 1-infected patients. Clin Vaccine Immunol 2009;16:11271131.

12. Cho YK, Sung H, Lee HJ, Joo CH, Cho GJ. Long-term intake of Korean red ginseng in HIV-1-infected patients: development of resistance mutation to zidovudine is delayed. Int Immunopharmacol 2001;1:1295-1305.

13. Bidawid S, Malik N, Adegbunrin O, Sattar SA, Farber JM. A feline kidney cell line-based plaque assay for feline calicivirus, a surrogate for Norwalk virus. J Virol Methods 2003;107:163-167.

14. Shibamoto T, Bjeldanes LF. Introduction to food toxicology. 2nd ed. Oxford: Academic Press, 2009.

15. D'Souza DH, Su X. Efficacy of chemical treatments against murine norovirus, feline calicivirus, and MS2 bacteriophage. Foodborne Pathog Dis 2010;7:319-326.

16. Iwasawa A, Niwano Y, Mokudai T, Kohno M. Antiviral activity of proanthocyanidin against feline calicivirus used as a surrogate for noroviruses, and coxsackievirus used as a representative enteric virus. Biocontrol Sci 2009;14:107-111.

17. Haidari M, Ali M, Ward Casscells S 3rd, Madjid M. Pomegranate (Punica granatum) purified polyphenol extract inhibits influenza virus and has a synergistic effect with oseltamivir. Phytomedicine 2009;16:1127-1136.

18. Su X, Howell AB, D'Souza DH. The effect of cranberry juice and cranberry proanthocyanidins on the infectivity of human enteric viral surrogates. Food Microbiol 2010;27:535-540.

19. Horm KM, D'Souza DH. Survival of human norovirus surrogates in milk, orange, and pomegranate juice, and juice blends at refrigeration $\left(4^{\circ} \mathrm{C}\right)$. Food Microbiol 2011;28:1054-1061.

20. Lane JA, Mehra RK, Carrington SD, Hickey RM. The food glycome: a source of protection against pathogen colonization in the gastrointestinal tract. Int J Food Microbiol 2010;142:1-13.

21. Sharon N, Lis H. Lectins--proteins with a sweet tooth: functions in cell recognition. Essays Biochem 1995;30:59-75.

22. Lee JH, Shim JS, Chung MS, Lim ST, Kim KH. Inhibi- 
Lee et al. Antiviral Activity of Korean Red Ginseng

tion of pathogen adhesion to host cells by polysaccharides from Panax ginseng. Biosci Biotechnol Biochem 2009; 73:209-212.

23. Rivera E, Ekholm Pettersson F, Inganas M, Paulie $\mathrm{S}$, Gronvik KO. The $\mathrm{Rb}_{1}$ fraction of ginseng elicits a balanced Th1 and Th2 immune response. Vaccine
2005;23:5411-5419.

24. Song X, Chen J, Sakwiwatkul K, Li R, Hu S. Enhancement of immune responses to influenza vaccine (H3N2) by ginsenoside Re. Int Immunopharmacol 2010;10:351356. 